\title{
Changes of physical and chemical characteristics during microbial composting of rice straw at various pH levels
}

\begin{abstract}
The physical and chemical parameters were monitored for seven weeks during Trichoderma/Aspergillus inoculated rice straw composting at various $\mathrm{pH}$ levels. Three treatments (A, B, and C) were inoculated with lignocellulolytic microbial consortium (Aspergillus niger, F44 and Trichoderma viride, F26) and three were un-inoculated (D, E, and F). $\mathrm{pH}$ of the starting materials was amended to 5.75 (A and D), 6.75 (B and E), and 7.75 ( $\mathrm{C}$ and $\mathrm{F}$ ) with either acetic acid or sodium hydroxide. Three typical phases of temperature were observed both in inoculated and un-inoculated treatments during composting: mesophilic phase, thermophilic phase, and followed by cooling and maturation phase. The bioconversion were maximum in Trichoderma/Aspergillus inoculated treatments within 1421 days as indicated by the profiles of electrical conductivity, bulk density, total carbon and nitrogen, and germination index. After day 21, the germination index of Trichoderma/Aspergillus inoculated treatment (B) without any $\mathrm{pH}$ amendment was increased to 74.5 indicating the maturity of compost and suitability for field application.
\end{abstract}

Keyword: Rice Straw; pH levels; Trichoderma/Aspergillus 